\title{
First Results from a Study of DIBs with Thousands of High-Quality Massive-Star Spectra
}

\author{
J. Maíz Apellániziz , A. Sota ${ }^{1}$, R. H. Barbá ${ }^{2}$, N. I. Morrell ${ }^{3}$, \\ A. Pellerin ${ }^{4}$, E. J. Alfaro ${ }^{1}$ and S. Simón-Díaz ${ }^{5}$ \\ ${ }^{1}$ Instituto de Astrofísica de Andalucía-CSIC, Spain \\ email: jmaiz@iaa.es \\ ${ }^{2}$ Universidad de La Serena, Chile \\ ${ }^{3}$ Las Campanas Observatory, Chile \\ ${ }^{4}$ Mount Allison University, Canada \\ ${ }^{5}$ Instituto de Astrofísica de Canarias, Spain
}

\begin{abstract}
We are using five different surveys to compile the largest sample of diffuse interstellar band (DIB) measurements ever collected. GOSSS is obtaining intermediate-resolution blue-violet spectroscopy of $\sim 2500$ OB stars, of which $60 \%$ have already been observed and processed. The other four surveys have already collected multi-epoch high-resolution optical spectroscopy of 700 OB stars with different telescopes, including the $9 \mathrm{~m}$ Hobby-Eberly Telescope in McDonald Observatory. Some of our stars are highly-extinguished targets for which no good-quality optical spectra have ever been published. For all of the targets in our sample we have obtained accurate spectral types, measured non-DIB ISM lines, and compiled information from the literature to calculate the extinction. Here we present the first results of the project, the properties of twenty DIBs in the 4100-5500 A range. We clearly detect a couple of previously elusive DIBs at $4170 \AA$ and $4591 \AA$; the latter could have coronene and ovalene cations as carriers.
\end{abstract}

Keywords. line: identification, line: profiles, surveys, stars: early-type, ISM: lines and bands

\section{The project}

The Galactic O-Star Spectroscopic Survey (GOSSS, Maíz Apellániz et al. 2011, Sota et al. 2011 ) is obtaining long-slit, $R \sim 2500$, blue-green spectroscopy of $\sim 2500$ massive stars in both hemispheres, including all known $\mathrm{O}$ stars with $B<12$ mag. Its main purpose is to characterize the O-star population in the solar neighborhood by providing accurate spectral types for all of the observed targets. We currently have spectra for 1592 stars and we plan to reach 2500 within two years.

Four other surveys are obtaining high-resolution spectroscopy of a subsample of the GOSSS stars with the purposes of doing detailed atmospheric modeling and calculating the orbits of the spectroscopic binaries. Three of those surveys, OWN (Barbá et al. 2010), IACOB (Simón-Díaz et al. 2011a, 2011b), and NoMaDS (Maíz Apellániz et al. 2012), have been described elsewhere. The fourth one, CAFÉ-BEANS (Calar Alto Fiberfed Echelle Binary Evolution Andalusian Survey, P.I.: Ignacio Negueruela), is obtaining multi-epoch $R=65000$ spectroscopy of northern stars using the CAFÉ spectrograph at the $2.2 \mathrm{~m}$ telescope at Calar Alto (Aceituno et al. 2012).

The original goal of these surveys was to study the stars but they also contain an unprecedented amount of information on optical ISM lines. That led us to start a 
Table 1. GOSSS extincted and reference stars used in this work.

\begin{tabular}{|c|c|c|c|}
\hline $\mid$ ID $\mid$ & Extincted star & \multicolumn{2}{|c|}{ | Spectral type $\mid E(B-J)$} \\
\hline 1 & Cyg OB2-12 & B5 Ia & 10.38 \\
\hline 2 & ALS 19626 & B0 Ia & 8.61 \\
\hline 3 & $\begin{array}{l}\text { 2MASS } \\
\text { J20333821+4041064 }\end{array}$ & B0 Ia & 8.33 \\
\hline 4 & ALS 21079 & O 7 Ib-II(f) & 7.64 \\
\hline 5 & Cyg OB2-22 B & O6 V((f)) & 7.56 \\
\hline 6 & Cyg OB2-22 A & O3 If* & 7.51 \\
\hline 7 & ALS 15114 & $\mathrm{O} 7.5 \mathrm{Vz}$ & 7.50 \\
\hline 8 & $\begin{array}{l}\text { 2MASS } \\
\text { J20344410+4051584 }\end{array}$ & O6.5 III(f) & 7.12 \\
\hline 9 & ALS 18747 & O5.5 Ifc & 5.94 \\
\hline $\mid$ ID $\mid$ & Reference star & \multicolumn{2}{|c|}{$\mid$ Spectral type $|E(B-J)|$} \\
\hline 1 & $\eta \mathrm{CMa}$ & B5 Ia & 0.37 \\
\hline 2 & HD 91969 & B0 Ia & 0.82 \\
\hline 3 & HD 91969 & B0 Ia & 0.82 \\
\hline 4 & HD 94963 & O 7 II & 0.68 \\
\hline 5 & HDE 303311 & $\mathrm{O} 6 \mathrm{~V}$ & 1.53 \\
\hline 6 & $\zeta$ Pup & $\mathrm{O} 4 \mathrm{I}(\mathrm{n}) \mathrm{f}$ & 0.07 \\
\hline 7 & HD 53975 & $\mathrm{O} 7.5 \mathrm{Vz}$ & 0.62 \\
\hline 8 & HD $152723 \mathrm{AaAb}$ & O $6.5 \mathrm{III}(\mathrm{f})$ & 1.45 \\
\hline 9 & HD 93632 & O5.5 Ifc & 2.33 \\
\hline
\end{tabular}

Table 2. Properties of the DIBs measured in this work using Gaussian fits. The first column gives the group ID (some DIBs were fitted together), the next two give the fit results, the fourth one the average equivalent widths of the used stars, and the last one the number of stars used.

\begin{tabular}{|r|r|r|r|r|}
\hline gr. & \multicolumn{1}{|c}{$\lambda_{\mathrm{c}}(\AA)$} & FWHM $(\AA)$ & EW $(\AA)$ & $n_{\mathrm{st}}$ \\
\hline 1 & $4179.48 \pm 0.61$ & $21.88 \pm 1.73$ & $0.62 \pm 0.14$ & 8 \\
2 & $4427.94 \pm 0.11$ & $24.15 \pm 0.30$ & $3.55 \pm 0.68$ & 9 \\
3 & $4501.67 \pm 0.09$ & $3.24 \pm 0.40$ & $0.17 \pm 0.04$ & 9 \\
4 & $4591.19 \pm 0.56$ & $24.95 \pm 1.58$ & $0.68 \pm 0.25$ & 6 \\
5 & $4726.70 \pm 0.05$ & $3.88 \pm 0.30$ & $0.28 \pm 0.05$ & 9 \\
6 & $4761.12 \pm 0.27$ & $19.72 \pm 1.06$ & $0.58 \pm 0.09$ & 9 \\
6 & $4762.36 \pm 0.09$ & $2.69 \pm 0.48$ & $0.10 \pm 0.01$ & 9 \\
6 & $4779.69 \pm 0.21$ & $5.48 \pm 0.57$ & $0.13 \pm 0.02$ & 9 \\
7 & $4879.83 \pm 0.16$ & $11.51 \pm 0.63$ & $0.43 \pm 0.07$ & 7 \\
7 & $4887.43 \pm 0.65$ & $39.75 \pm 1.14$ & $2.16 \pm 0.36$ & 7 \\
8 & $4963.85 \pm 0.15$ & $2.62 \pm 0.66$ & $0.06 \pm 0.02$ & 8 \\
9 & $4984.59 \pm 0.23$ & $1.33 \pm 1.29$ & $0.02 \pm 0.01$ & 8 \\
10 & $5109.66 \pm 0.43$ & $14.91 \pm 1.34$ & $0.23 \pm 0.08$ & 6 \\
11 & $5155.99 \pm 0.49$ & $15.97 \pm 1.50$ & $0.14 \pm 0.03$ & 7 \\
12 & $5236.29 \pm 0.24$ & $2.27 \pm 0.77$ & $0.06 \pm 0.02$ & 5 \\
12 & $5245.43 \pm 0.57$ & $7.26 \pm 1.56$ & $0.08 \pm 0.03$ & 5 \\
13 & $5363.52 \pm 0.13$ & $2.74 \pm 0.55$ & $0.07 \pm 0.01$ & 7 \\
14 & $5449.83 \pm 0.22$ & $14.06 \pm 0.61$ & $0.48 \pm 0.13$ & 8 \\
15 & $5487.23 \pm 0.12$ & $6.63 \pm 0.33$ & $0.35 \pm 0.05$ & 6 \\
15 & $5494.29 \pm 0.26$ & $1.90 \pm 0.96$ & $0.04 \pm 0.01$ & 6 \\
\hline
\end{tabular}

parallel project to obtain and process such information. In this first analysis we study the properties of the DIBs seen in the GOSSS data.

\section{4100-5500 ̊̊ DIB properties}

We selected 9 stars observed by GOSSS with high extinction, good S/N spectra, and coverage of the full 3900-5500 A range (Table 1). For each star we selected a corresponding GOSSS star with low extinction and similar spectra type in order to apply the traditional pair method, in which the low-extinction spectrum is subtracted from the high-extinction one in order to eliminate the stellar contribution. The spectra were put in the ISM reference system using the CaII $\lambda 3934$ line. The average profile was then calculated for 

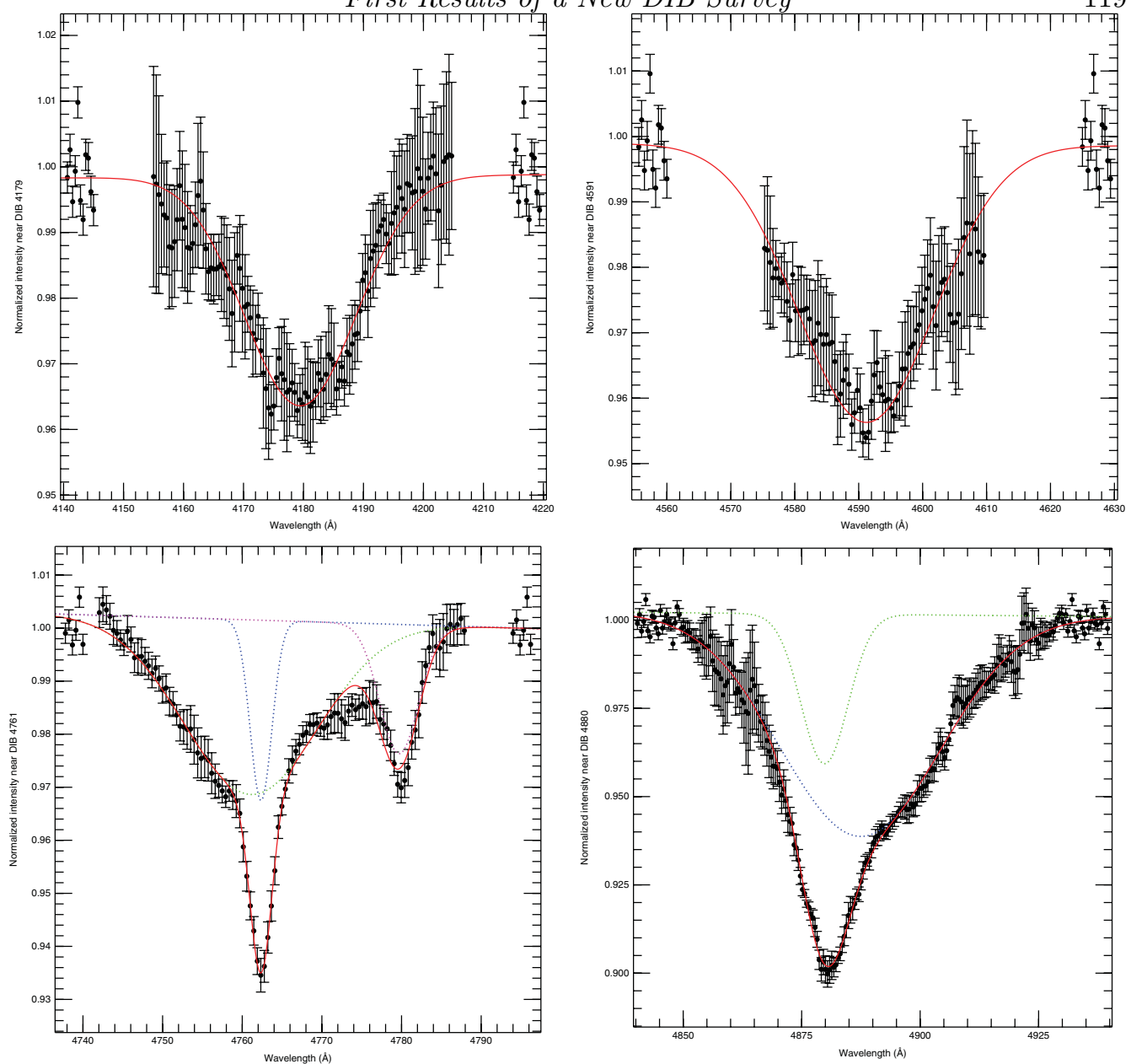

Figure 1. Gaussian fits for DIB groups 1, 4, 6, and 7. Error bars show the standard deviation of the average profile normalized by EW. Dotted lines are used for individual components.

each DIB by selecting the stars in the sample with the largest EWs, normalizing by it, and calculating the mean and standard deviation of the spectra at each wavelength. The result was fit using either Gaussian and Lorentzian profiles (multiple in the case where DIBs overlap so they must be fitted in groups). Finally, the instrumental width was subtracted to the fit FWHM. Results for the Gaussian fits are shown in Table 2.

- We clearly detect the seldom seen $4179 \AA$ broad DIB (Knoechel \& Moffat 1982, Jenniskens \& Désert 1994; Fig. 1).

- The broad intense $4428 \AA$ DIB is better fit by a Lorentzian than a Gaussian (see Snow et al.2002). However, the differences are small and the broad Lorentzian wings make rectification difficult in practice, so Gaussian fits are less noisy for most data.

- We detect the previously elusive $4591 \AA$ broad DIB, which could be produced by coronene $\left(\mathrm{C}_{24} \mathrm{H}_{12}\right)$ and ovalene $\left(\mathrm{C}_{32} \mathrm{H}_{14}\right)$ cations (Ehrenfreund et al. 1995, Fig. 1).

- The $4770 \AA$ region requires a fit with two narrow and one broad DIBs (Fig. 1).

- The $4880 \AA$ A region requires a fit with one broad and one intermediate DIBs (Fig. 1).

- The $5110 \AA$ AIB shows a possible additional component in its left wing.

- There may be a broad component around the $5364 \AA$ DIB (not measured). 
- At this resolution the two narrow DIBs to the left and right of He II $\lambda 5412$ are blended with the stellar line and are not analyzed here.

- The $5240 \AA$ A region shows two clearly separated components at this resolution.

- The $5490 \AA$ region shows two clearly separated components at this resolution.

\section{References}

Aceituno, J., et al. 2012, A\&A 552, A31

Barbá, R. H., et al. 2010, Revista mexicana de astronomía y astrofísica (serie de conf.) 38, 30 Ehrenfreund, P., et al. 1995, A\&A 299, 213

Jenniskens, P. \& Désert, F.-X. 1994, A\&AS 106, 39

Knoechel, G. \& Moffat, A. F. J. 1982, A\&SA 110, 263

Maíz Apellániz, J., et al. 2011, Highlights of Spanish Astrophysics VI, 467

Maíz Apellániz, J., et al. 2012, ASP Conference Series 465, 484

Simón-Díaz, S., Castro, N., García, M., \& Herrero, A. 2011a, IAU Symposium 272, 310

Simón-Díaz, S., et al. 2011b, arXiv 1109.2665

Snow, T. P., Zukowski, D., \& Massey, P. 2002, ApJ 578, 877

Sota, A., et al. 2011, ApJS 193, 24 